\title{
Studienübergreifende Harmonisierung datenschutzrechtlicher und ethischer Gesichtspunkte in Patientenunterlagen: Eine Praxisanalyse
}

\author{
Monika Kraus, Matthias Nauck, Dana Stahl, Arne Blumentritt, \\ Gabriele Anton, H.-Erich Wichmann und Annette Peters
}

M. Kraus $(\square) \cdot$ G. Anton $\cdot$ H.-E. Wichmann $\cdot$ A. Peters

Institut für Epidemiologie, Helmholtz Zentrum München, Deutsches Forschungszentrum für Gesundheit und Umwelt, München, Deutschland

E-Mail: monika.kraus@helmholtz-muenchen.de

G. Anton

E-Mail: gabriele.anton@helmholtz-muenchen.de

H.-E. Wichmann

E-Mail: wichmann@helmholtz-muenchen.de

A. Peters

E-Mail: peters@helmholtz-muenchen.de

M. Nauck

Institut für Klinische Chemie und Labormedizin, Universitätsmedizin Greifswald, Greifswald, Deutschland

E-Mail: matthias.nauck@med.uni-greifswald.de

D. Stahl · A. Blumentritt

Unabhängige Treuhandstelle, an der Universitätsmedizin Greifswald, Greifswald, Deutschland E-Mail: Dana.Stahl@uni-greifswald.de

A. Blumentritt

E-Mail: arne.blumentritt@uni-greifswald.de

A. Peters

Institute for Medical Information Processing, Biometry and Epidemiology,

Ludwig-Maximilians-Universität München, München, Deutschland

(C) Der/die Autor(en) 2022

G. Richter et al. (Hrsg.), Datenreiche Medizin und das Problem der Einwilligung,

https://doi.org/10.1007/978-3-662-62987-1_9 


\section{$1 \quad$ Einleitung}

In diesem Erfahrungsbericht beschreiben wir einen konkreten Anwendungsfall der Nutzung und Einreichung einer studien- und standortübergreifenden Infrastruktur bei zahlreichen Ethikkommissionen unter den Bedingungen, die kurz vor und nach vollumfänglichem verbindlich werden der DSGVO gegeben waren. Dieser Anwendungsfall hat unter anderem die studienübergreifende Harmonisierung datenschutzrechtlicher und ethischer Gesichtspunkte in Patientenunterlagen zum Ziel. Alle Daten sind aus der praktischen Arbeit entnommen. Einige davon sind laufend in aktueller Form im Einsatz zur regelgerechten Durchführung einer Daten- und Biomaterial-Infrastruktur im Kontext klinischer Studien - der klinisch-wissenschaftlichen Forschungsplattform des DZHK e. V. (DZHK e. V. 2017, 2019b).

Vom DZHK e. V. überwiegend finanzierte multizentrische klinische Studien nutzen verpflichtend die klinische Forschungsplattform (Abb. 1) zur Erhebung und Speicherung von medizinischen Daten in zentralen Datenbanken und zum Management der zweckoffenen Basis-Biomaterialsammlung. Zu diesem Zweck wird die Software secuTrial ${ }^{\circledR}$ genutzt, die von der Universitätsmedizin Göttingen als einem der Partner der klinischen Forschungsplattform betrieben wird. Für das Bilddatenmanagement steht Trial Connect zur Verfügung sowie die Software CentraXX für das Biomaterialmanagement.

Das Ziel dieser Forschungsplattform ist die Sekundärnutzung standardisiert erhobener Studiendaten. Das zentrale Identitätsmanagement als Pseudonymisierungsdienst sowie das Consent-Management erfolgen durch eine unabhängige Treuhandstelle. Diese hat niemals Zugang zu den medizinischen Patientendaten so wie die Komponenten der

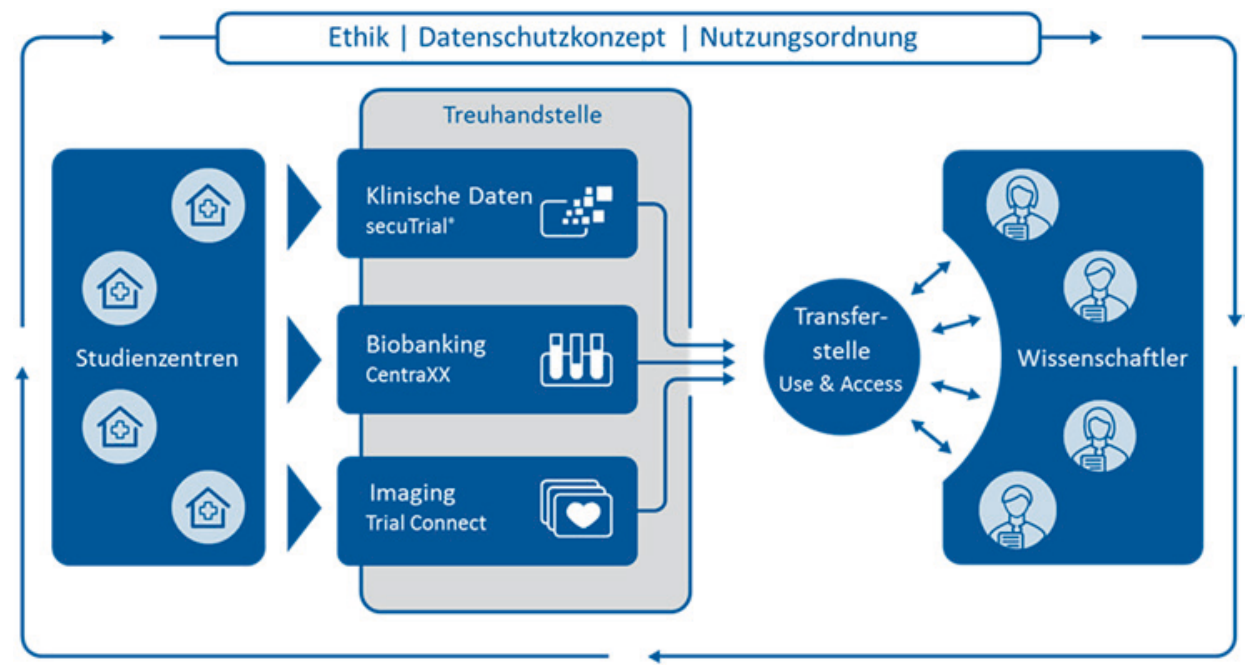

Abb. 1 Aufbau und Aufgabenbereiche der einzelnen IT-Partner innerhalb der Klinischen Forschungsplattform des DZHK. Der Bereich „Ethik“ wird durch das Ethik-Projekt als Teil der Forschungsplattform vertreten 
Datenhaltung niemals Zugang zu identifizierenden Patientendaten haben. Auf diese Weise ist eine strenge Aufrechterhaltung der informationellen Gewaltenteilung gewährleistet. Um die Abbildung dieses Systems in den Patientenunterlagen umzusetzen und die Inhalte der Patientenunterlagen für die Treuhandstelle elektronisch abbildbar zu machen, vervollständigt das Ethik-Projekt als Teilprojekt die klinische Forschungsplattform. Flankiert wird die Forschungsplattform von Dokumenten und Prozessen, die der Einhaltung von regulierenden Grundsätze dienen. Dabei handelt es sich um SOPs, eine Nutzungsordnung, ein Datenschutzkonzept, ein Ethik-Konzept und weitere Use and Access Prozesse. Diese regulieren sowohl die Seite der Daten- und Biomaterialerhebung als auch die Seite der Herausgabe der dadurch entstandenen Ressourcen für Forschungszwecke. Die zur Verfügung gestellten IT-Systeme werden bereits von 19 multizentrischen klinischen Studien in über 100 Studienzentren im Inland sowie weiteren Studienzentren im europäischen Ausland genutzt.

Ein Erklärvideo steht unter folgender Adresse zur Verfügung https://www.youtube. $\mathrm{com} /$ watch? $\mathrm{v}=270$ VuBvzcj0\&feature=youtu.be (DZHKe. V. 2019a).

Für die regulierte, aber zweckoffene Nutzung von Bioproben und Daten wurde ein Antrags- und Zugangsprozess (Scheel et al. 2019; Zeller et al. 2014; DZHKe. V. 2018) $\mathrm{zu}$ den gespeicherten medizinischen Daten und Bioproben für weitere medizinische Forschungsprojekte implementiert.

Die Basis für die Umsetzung des Systems bilden die Nutzungsordnung, das Datenschutzkonzept und ein Ethik-Konzept. Letzteres enthält außerdem Muster-Patienteninformationsund Einwilligungsunterlagen (Patient Information and Consent „PIC“) mit bereits integriertem Informationstext zur Datenspeicherung in der Forschungsplattform des DZHK und der breiten Nutzung dieser für wissenschaftliche Forschungsprojekte. Teil der Forschungsplattform ist außerdem die zweckoffene Basis-Biomaterialsammlung des DZHK. Die Informationen zur Datenspeicherung und Biomateriallagerung sowie die Voraussetzungen zur Nutzung dieser für Forschungsprojekte werden in den Patientenunterlagen abgebildet, um dem Patienten dies im Sinne einer informierten Einwilligung transparent darzustellen. Das führt zur Notwendigkeit der Harmonisierung von Patienteninformationsund Einwilligungsunterlagen und kann gewährleistet werden, indem die Muster-PICs des DZHK Ethik-Konzeptes genutzt und studienspezifisch angepasst werden. Aufgabe des verpflichtend hinzuzuziehenden Ethik-Projektes ist es, zu überprüfen, ob alle Voraussetzungen zur Nutzung der klinischen Forschungsplattform in den Patientenunterlagen abgebildet sind sowie die studienübergreifenden Strukturen und Prozesse bei Nachfragen durch Ethikkommissionen und andere involvierte Parteien zu erklären. Um die Umsetzung der genannten Inhalte zu gewährleisten, unterstützt das Ethik-Projekt die Studien außerdem bei Ethikeinreichungen.

Entsprechend ergänzte Patienteninformations- und Einwilligungsunterlagen (PIC) der DZHK-Studien wurden seit 2013 bereits von über 50 Ethikkommissionen deutschlandweit positiv begutachtet.

Die PICs der Basis-Biomaterialsammlung werden in der Regel als ergänzende Dokumente zur klinischen Studie im gleichen Einreichungsprozess den jeweils beteiligten Ethikkommissionen zur Verfügung gestellt. 


\section{$2 \quad$ Ziel}

Das Ethik-Projekt als Teilprojekt der klinischen Forschungsplattform ist täglich mit den Anforderungen konfrontiert, die eine deutschlandweit heterogene und derzeit zersplitterte Landschaft der ethischen und datenschutzrechtlichen Bewertung von klinischen Studien hervorruft. In diesem regulatorisch anspruchsvollen Umfeld ist es eine besondere Herausforderung ein standardisiertes Datenmanagement- und ZugangsSystem zu unterstützen, das eben diese Studien überspannt und sich auf mehr als 100 Studienzentren erstreckt. Das derzeitige Bewertungssystem führt dazu, dass multizentrische Forschungsvorhaben bei multiplen Stellen eingereicht werden müssen (v. a. bei einer Beratungspflicht nach Berufsrecht). Sich daraus ergebende zahlreiche lokale Änderungsforderungen und -wünsche stellen ein teils standardisiertes, teils harmonisiertes System wie das hier beschriebene vor schwer bis kaum lösbare Herausforderungen. Übergreifende Strukturen wie dieses scheinen an der unterschiedlichen Bewertung von Vorhaben durch Ethikkommissionen und unterschiedlichen Auslegungen des Datenschutzes zu scheitern.

Obwohl i. d. R. individuelle lokale Lösungen mit den zuständigen Ethikkommissionen vor Ort gefunden werden können, ist dieser Prozess für eine Struktur wie der des DZHK extrem aufwendig. Um diesen Eindruck mit qualitativen und quantitativen Untersuchungen aus der Alltags-Praxis des Ethik-Projektes zu untermauern wurden Analysen der Einreichungsprozesse aus den Jahren 2017 und 2018 sowie Inhalte und Anzahl der von Ethik-Projekt und Treuhandstelle verwalteten Einwilligungsversionen der klinischen Studien und der Biomaterialsammlung durchgeführt.

\section{Analysen und Ergebnisse}

Im ersten Schritt wurden die Inhalte von Ethikvoten anhand einer Verschlagwortung und Kategorisierung strukturiert. Die Vielzahl an unterschiedlichen Fragestellungen führte zur Idee eine Analyse durchzuführen, welche Anpassungen von Standardprozessen als Reaktionen auf Rückmeldungen von Ethikkommissionen bis November 2019 notwendig waren. Bezüglich einzelner Standardprozesse, nämlich der Speicherzeitbegrenzung, des Widerrufsprozesses und der Weitergabe von Daten für die Forschung liegen für den gemeinsamen Betrieb der Forschungsplattform die Information in textbasierten Tabellen vor. Diese wurden für die Analysen extrahiert und betrachtet. Anschließend wurden die Anpassungen und daraus folgende Konsequenzen untersucht.

Woraufhin von Interesse war, wie viele Versionen von Einwilligungsunterlagen in Folge der Änderungen durch das Ethik-Projekt und die Treuhandstelle für die 19 Studien des DZHK und die Biomaterialsammlung verwaltet werden müssen. 


\subsection{Auswertung Ethikvoten}

\subsubsection{Methode}

Aufgrund der Komplexität eines derartigen Systems beantwortete das Ethik-Projekt bei 17 von 19 Studien vor der Ausstellung eines finalen Ethik-Votums Anfragen der erstberatenden oder federführenden Ethikkommissionen, um deren Zustimmung zur Nutzung der klinischen Forschungsplattform des DZHK zu erhalten. Um einen Überblick darüber zu gewinnen, welche Inhalte bei diesen deutschlandweiten Einreichungen mit Ethikkommissionen diskutiert wurden, wurden die Inhalte von 43 Ethikvoten aus den Jahren 2017 und 2018 vom Ethik-Projekt des DZHK ausgewertet und kategorisiert (Anzahl bearbeitete Voten 2017: 21; 2018: 22).

Bei den Ethikvoten handelte es sich um Schreiben von Ethikkommissionen, die versehen waren mit Nachfragen $\mathrm{zu}$ die klinische Forschungsplattform betreffenden Prozessen. Ethikvoten ohne Anfragen zur Forschungsplattform wurden nicht berücksichtigt.

Die analysierten Ethikvoten stammten von 16 unterschiedlichen Ethikkommissionen und betrafen 11 verschiedene multizentrische DZHK Studien. Alle Studien nutzen die klinische Forschungsplattform des DZHK in vollem Umfang.

In einem ersten Schritt der Analyse wurden Schlagworte auf Basis folgender Kriterien vergeben:

- Jeder Absatz der ein Thema umschreibt ergibt ein Schlagwort

- Klar formulierte Umsetzungshinweise ergeben jeweils ein eigenes Schlagwort

Beispiel:

„Warum müssen die Daten der randomisierten Hauptstudie in personenbeziehbarer Form auf unbestimmte Zeit gespeichert werden (siehe Datenschutzerklärung, Einwilligungserklärung Seite 2)? Nach dem Prinzip der Datensparsamkeit müssen Daten anonymisiert werden, sobald der Forschungszweck dies zulässt.“

$\rightarrow$ Vergabe des Schlagwortes Speicherbegrenzung und Einordnung in die Kategorie 2

Es wurden 43 Ethikvoten inhaltlich analysiert, indem Schlagworte nach den oben definierten Kriterien vergeben wurden. Die Gesamtzahl der vergebenen Schlagworte betrug 173, das heißt ein Schreiben wurde durchschnittlich mit vier Schlagworten versehen. Die Anzahl der Schlagworte war allerdings sehr heterogen verteilt zwischen den Ethikvoten (zwischen einem und 11 Schlagworten).

Anschließend wurden die Schlagworte in sechs Kategorien zusammengefasst, die inhaltlich und bezüglich der formulierten Antworten in Ethikvoten häufig in Zusammenhang standen.

Dies umfasste jeweils folgende Bereiche, die eine Kategorie ergaben (Tab. 1). 
Tab. 1 Beschreibung der Kategorien und deren Inhalte

\begin{tabular}{|c|c|c|c|c|c|}
\hline \multirow[t]{2}{*}{ Kategorie } & \multirow[t]{2}{*}{ Bezeichnung } & \multirow[t]{2}{*}{ Inhalte } & \multicolumn{3}{|c|}{ Anzahl Schlagworte } \\
\hline & & & gesamt & 2017 & 2018 \\
\hline 1 & $\begin{array}{l}\text { Datenverarbeitung } \\
\text { in der Klinischen } \\
\text { Forschungsplattform }\end{array}$ & $\begin{array}{l}\text { Struktur der klinischen } \\
\text { Forschungsplattform/ } \\
\text { Informationelle Gewalten- } \\
\text { teilung/Anonymisierung/ } \\
\text { Pseudonymisierung }\end{array}$ & 22 & 15 & 7 \\
\hline 2 & $\begin{array}{l}\text { Speicherdauer und } \\
\text { Zweckbestimmung }\end{array}$ & $\begin{array}{l}\text { Daten-/Biomaterialspeicher- } \\
\text { dauer, Zweckbestimmung }\end{array}$ & 26 & 12 & 14 \\
\hline 3 & $\begin{array}{l}\text { Widerruf und Daten- } \\
\text { löschung }\end{array}$ & $\begin{array}{l}\text { Widerrufsprozesse, Prozess der } \\
\text { Datenlöschung }\end{array}$ & 21 & 11 & 10 \\
\hline 4 & $\begin{array}{l}\text { Governance und Nach- } \\
\text { nutzung, Rückmeldung }\end{array}$ & $\begin{array}{l}\text { Governance der Daten- und } \\
\text { Biomaterialsammlung, Daten- } \\
\text { weitergabe, Nachnutzung von } \\
\text { Daten und Biomaterialien, } \\
\text { Zufallsbefunde, genetische } \\
\text { Untersuchungen }\end{array}$ & 21 & 14 & 7 \\
\hline 5 & $\begin{array}{l}\text { EU-DSGVO und Dritt- } \\
\text { länder }\end{array}$ & $\begin{array}{l}\text { EU-DSGVO } \\
\text { Weitergabe von Daten in } \\
\text { unsichere Drittländer }\end{array}$ & 35 & 9 & 26 \\
\hline 6 & $\begin{array}{l}\text { Prozess der Ein- } \\
\text { reichung (sowie } \\
\text { Formatierung und } \\
\text { Formulierung) }\end{array}$ & $\begin{array}{l}\text { Gesonderte Unterlagen oder } \\
\text { gesonderte Ethikeinreichung } \\
\text { Biomaterialsammlung, Muster- } \\
\text { texte, „Prozess Ethikein- } \\
\text { reichung“ }\end{array}$ & 36 & 21 & 15 \\
\hline 7 & keine & $\begin{array}{l}\text { Nicht den Kategorien } 1 \text { bis } 6 \\
\text { zuzuordnen }\end{array}$ & 12 & 6 & 6 \\
\hline \multicolumn{3}{|l|}{ Gesamt } & 173 & 88 & 85 \\
\hline
\end{tabular}

\subsubsection{Ergebnis}

Bei Betrachtung der Jahre 2017 und 2018 zusammen in Abb. 2 (lila Balken) zeigt sich eine relativ gleichmäßige Verteilung der Schlagwörter auf die Kategorien 1 bis 4 mit jeweils 21 bis 26, sowie die Kategorien 5 und 6 mit 35 bzw. 36 Schlagwörtern (siehe auch Tab. 1 Anzahl Schlagworte ges.).

Ein anderes Bild ergibt sich nach Aufteilung der Schlagwortvergabe nach Jahren:

Eine Verschiebung von eher generellen Fragen bezüglich der Prozesse wie Datenmanagement (Kategorie 1), Governance/Nachnutzung (Kategorie 4) und Einreichungsprozessen (Kategorie 6) im Jahr 2017 (Abb. 2, blaue Balken), hin zu Fragen und Anmerkungen, die spezifischer die DSGVO betreffen (Kategorie 5) im Jahr 2018 (Abb. 2, rote Balken), ist deutlich erkennbar. Zu bemerken ist auch, dass Schlagworte zu Speicherdauer und Zweckbestimmung (Kategorie 2) sowie zu Widerruf und Datenlöschung (Kategorie 3) in den Voten der zwei Jahre etwa gleich häufig vergeben wurden. 


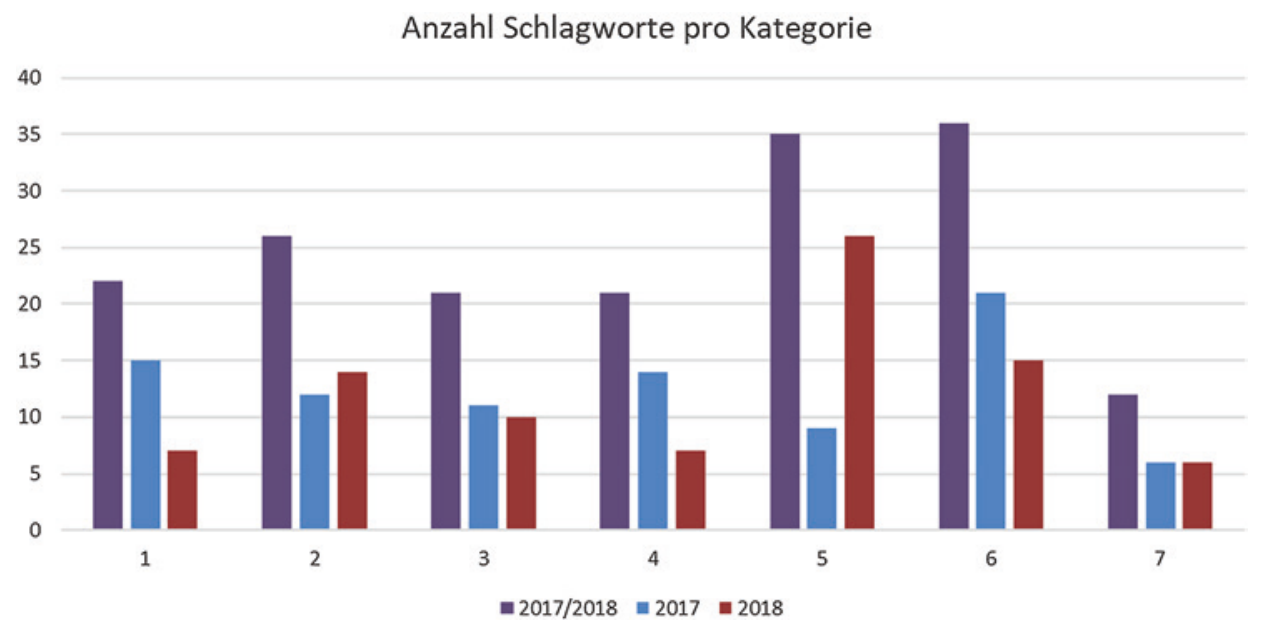

Abb. 2 Anzahl der Schlagworte pro Kategorie. Die x-Achse stellt die Kategorien 1-7 dar, auf der $\mathrm{y}$-Achse ist die absolute Anzahl der vergebenen Schlagwörter pro Kategorie angegeben

\subsection{Betrachtung von Prozessanpassungen}

Als Folge der zahlreichen Rückfragen von Ethikkommissionen und Forderungen nach Anpassungen war es mitunter notwendig, individuelle Lösungen mit einzelnen Ethikkommissionen zu finden. Das führte in nicht wenigen Fällen zu Formulierungsänderungen in Patientenunterlagen, was wiederum Änderungen in den Prozessen der klinischen Forschungsplattform nach sich zog.

Unter einem Prozess ist in diesem Zusammenhang die regelkonforme technische und organisatorische Umsetzung einzelner Bearbeitungsschritte zu verstehen, die zu einem definierten Ergebnis führt. (z.B. die Löschung von Daten zu einem festgelegten Zeitpunkt).

Daher wurde eine Analyse bezüglich ausgewählter notwendiger Prozessanpassungen durchgeführt, betreffend das Daten- und Biomaterialmanagement.

\subsubsection{Vorgehen}

Beispielhaft wurden die Inhalte der Patientenunterlagen aus 19 DZHK-Studien (Stand November 2019) bezüglich der Formulierungen zu den Punkten

- Speicherzeitbegrenzung (s. Tab. 2)

- Widerruf (s. Tab. 3) und Recht auf Löschung (s. Tab. 4)

- Weitergabe von Daten für die Forschung (s. Tab. 5)

überprüft. Die Analysen wurden aus bereits bestehenden Tabellen erstellt, die für den regulären Betrieb der klinischen Forschungsplattform als gemeinsame Schnittstelle des Ethik-Projektes mit der Treuhandstelle dienen. 
Für die Darstellung wurden sie in die Übersichtstabellen 2 bis 5 überführt.

Der Standardprozess (nicht farblich hervorgehoben) wurde den aus inhaltlichen Anpassungen folgenden Prozessanpassungen gegenübergestellt (farblich hervorgehoben in Tab. 2 bis 5).

\subsubsection{Ergebnisse}

Im Folgenden werden die Ergebnisse bezüglich der unter Abschn. 3.2.1 genannten Punkte einzeln betrachtet.

\section{Speicherzeitbegrenzung}

Das standardmäßig etablierte Vorgehen des DZHK beinhaltet die unbefristete Speicherung von standardisiert erhobenen Patientendaten und die unbefristete Lagerung von standardisiert gewonnenen und prozessierten Bioproben, bis diese aufgebraucht sind. Die Einwilligung dazu bleibt bis zu einem Widerruf des Patienten bestehen. Das Vorgehen ist mit den notwendigen Zugangsbestimmungen zur Nachnutzung (verbindliche Nutzungsordnung, Zugangskomitee, Einbeziehung einer Ethikkommission für Nachnutzungsprozesse) im Sinne des Broad Consent von Seiten des Arbeitskreises medizinischer Ethikkommissionen (AKEK) seit mehreren Jahren etabliert und wurde in der Handreichung für Ethikkommissionen niedergeschrieben (Empfehlung für die Bewertung forschungsbezogener Biobanken durch Ethik-Kommissionen 2016). Das DZHK führt dieses Vorgehen seit 2013 durch.

Dem Standardprozess entgegenstehende inhaltliche Anpassungen in Patientenunterlagen nach Forderungen und/oder Hinweisen von Ethikkommissionen und als Konsequenz daraus erfolgte Prozessanpassungen werden in Tab. 2 dargestellt:

Tab. 2 Auswertung der Inhalte von 75 PICs aus 19 DZHK Studien (Stand Nov 2019) zum Punkt Speicherzeitbegrenzung

\begin{tabular}{l|l}
\hline $\begin{array}{l}\text { Inhaltliche Anpassungen in Patientenunterlagen } \\
\text { nach Forderungen und Hinweisen von Ethik- } \\
\text { kommissionen }\end{array}$ & $\begin{array}{l}\text { Prozessanpassungen als Konsequenz aus den } \\
\text { Inhaltlichen Anpassungen in Patientenunter- } \\
\text { lagen }\end{array}$ \\
\hline $\begin{array}{l}\text { Speicherung formuliert für „....mindestens 10 } \\
\text { Jahre... }\end{array}$ & Keine Anpassung erforderlich \\
\hline $\begin{array}{l}\text { Speicherung auf 10 Jahre begrenzt } \\
\begin{array}{l}\text { Speicherung bis 15 Jahre nach Ende der Studie, } \\
\text { dann Löschen sofern nicht optional eine } \\
\text { unbefristete Speicherung eingewilligt wurde }\end{array}\end{array}$ & $\begin{array}{l}\text { 15 Jahre nach Ende der Studie Löschung, } \\
\text { verknüpfung mit optionaler unbefristeter } \\
\text { Speicherung }\end{array}$ \\
\hline $\begin{array}{l}\text { Speicherung bis eine weitere Verwendung nicht } \\
\text { mehr beabsichtigt ist }\end{array}$ & Überprüfung der Daten notwendig, wann? \\
\hline $\begin{array}{l}\text { Lagerung von Biomaterialien auf 25 Jahre } \\
\text { begrenzt }\end{array}$ & $\begin{array}{l}\text { Biomaterialien müssen nach 25 Jahren ver- } \\
\text { nichtet werden }\end{array}$ \\
\hline
\end{tabular}


Zur Erreichung eines uneingeschränkt positivem Ethikvotums für alle Standorte und Studienzentren wurden folgende Prozessanpassungen durchgeführt:

- die Löschung von Daten 10 bzw. 15 Jahre nach Ende der Studie sowie die Vernichtung von Biomaterialien 25 Jahre nach Ende der Studie wurde hinterlegt.

- eine Speicherung für ,,mindestens 10 Jahre“ verlangt keine Prozessänderung, da es sich um eine reine Formulierungsänderung handelt. Es ist kein Höchstzeitraum angegeben.

\section{Widerruf}

Der Standardprozess des Widerrufs stellt sich im DZHK folgendermaßen (in Teilprozesse untergliedert) dar:

1. Vernichtung der Bioproben

2. Anonymisierung von Daten

3. Sperrung von Daten für die Weitergabe an Forschungsprojekte

Standardmäßig wird bei einem vollständigen Widerruf der Teilnahme eines Patienten an einer DZHK-Studie und an der DZHK Basis-Biomaterialsammlung die Vernichtung der Bioproben durchgeführt. Auch die Anonymisierung von Daten und die Sperrung dieser für die Weitergabe an Forschungsprojekte (laut Nutzungsordnung des DZHK) wird umgehend initiiert. Eine Nutzung ist damit nur noch für die Qualitätssicherung erlaubt. Es ist vorgesehen, anonymisierte Daten in regelmäßigem Turnus zu überprüfen und, wenn diese nicht mehr für die Qualitätssicherung notwendig sind, zu löschen.

Tab. 3 Auswertung der Inhalte von 75 PICs aus 19 DZHK Studien (Stand Nov 2019) zum Punkt Widerruf

\begin{tabular}{l|l}
\hline $\begin{array}{l}\text { Inhaltliche Anpassungen in Patientenunterlagen } \\
\text { nach Forderungen und Hinweisen von Ethik- } \\
\text { kommissionen }\end{array}$ & $\begin{array}{l}\text { Prozessanpassungen als Konsequenz aus den } \\
\text { Inhaltlichen Anpassungen in Patientenunter- } \\
\text { lagen }\end{array}$ \\
\hline AMG & $\begin{array}{l}\text { keine Anonymisierung, ggf. muss ein Teil der } \\
\text { Bioproben erhalten bleiben }\end{array}$ \\
\hline $\begin{array}{l}\text { Patient kann optional angeben ob Daten } \\
\text { gelöscht oder anonymisiert werden }\end{array}$ & $\begin{array}{l}\text { Spezifizierte Übermittlung des Widerrufes, } \\
\text { nämlich mit Angabe welche Version durch den } \\
\text { Patienten ausgewählt wurde }\end{array}$ \\
\hline $\begin{array}{l}\text { Datenweitergabe ist auch nach Widerruf in } \\
\text { anonymisierter Form möglich }\end{array}$ & $\begin{array}{l}\text { Keine Sperrung anonymisierter Daten für die } \\
\text { Weitergabe (wird derzeit nicht durchgeführt) }\end{array}$ \\
\hline $\begin{array}{l}\text { Datenweitergabe ist, als Option für den } \\
\text { Patienten, nach Widerruf in anonymisierter } \\
\text { Form möglich }\end{array}$ & $\begin{array}{l}\text { spezifizierte Übermittlung des Widerruf, näm- } \\
\text { lich mit Angabe welche Version durch den } \\
\text { Patienten ausgewählt wurde }\end{array}$ \\
\hline
\end{tabular}


Neben diesem Standardvorgehen bestehen folgende Versionen durch Änderung von Formulierungen in Patientenunterlagen:

Bei AMG-Studien wurde bisher von der Formulierung abgewichen. Hier wird aufgrund der gesetzlichen Archivierungs-/Nachweispflicht von einer Anonymisierung abgesehen (AMG i.V.m GCP-V §13 und ICH-GCP § 8). Dies ist bei vier DZHK-Studien der Fall und soll nicht weiter diskutiert werden, da es sich um eine arzneimittelrechtliche Vorsichtsmaßnahme handelt, die der Patientensicherheit dient. Inwiefern es diesbezüglich im Zuge der weiteren Etablierung der DSGVO Änderungen geben wird, ist uns derzeit noch nicht bekannt.

Bei Nicht-AMG Studien mussten folgende inhaltlichen Änderungen durchgeführt werden, um ein uneingeschränkt positives Ethikvotum zu bekommen:

- Inkludierung einer optionalen Angabe für eine Entscheidung durch den Patienten, ob Daten gelöscht oder anonymisiert werden sollen.

- Inkludierung einer optionalen Angabe für eine Entscheidung durch den Patienten, ob eine Datenweitergabe auch nach Widerruf in anonymisierter Form möglich ist

Einige Unterlagen enthalten außerdem folgenden Inhalt:

- Die Datenweitergabe ist nach Widerruf in anonymisierter Form möglich

Sobald in den Unterlagen Auswahlmöglichkeiten für den Patienten enthalten sind, ist eine spezifizierte Übermittlung des Widerrufes, nämlich mit Angabe, welche Version durch den Patienten ausgewählt wurde, notwendig.

\section{Recht auf Löschung}

Im Rahmen der Widerrufsmöglichkeiten wurde auch das seit Einführung der DSGVO mit größerem Gewicht bedachte Recht auf Löschung betrachtet. Seit Inkrafttreten der DSGVO ist jedem Patienten ein Recht auf Löschung seiner personenbezogenen Daten (,Recht auf Vergessenwerden“) einzuräumen (DSGVO Art.17, hier besonders zu beachten Art.17(1)b)).

Jedoch wurden Einschränkungen des Rechts auf Löschung bereits in der Vergangenheit bezüglich der Verhältnismäßigkeit und des Aufwandes der Löschung formuliert (Kai 2020). Auch wurden Formulierungen zu Ausnahmen von der Löschpflicht bei Datenspeicherung und -nutzung aus Gründen des öffentlichen Interesses im Bereich der öffentlichen Gesundheit und zu wissenschaftlichen Forschungszwecken in der DSGVO niedergeschrieben (DSGVO Art.17(3)c und d).

Von Seiten des Ethik-Projektes wurden die Studien unterstützt, schnellstmöglich die Betroffenenrechte nach DSGVO in den PICs zu ergänzen. Die Formulierung wurde allerdings aufgrund der zum Zeitpunkt der Erstellung/ Überarbeitung der Patientenunterlagen noch unklaren Interpretation der Gesetzestexte unterschiedlich gehandhabt, was zu unterschiedlichen Formulierungen des Rechtes der Datenlöschung in den Patientenunterlagen der DZHK-Studien führte.

Der Prozess der Datenlöschung wird derzeit individuell nach formloser Meldung des Wunsches des Patienten an Studienzentrum oder Treuhandstelle durchgeführt. 
Tab. 4 Auswertung der Inhalte von 75 PICs aus 19 DZHK Studien (Stand Nov 2019) zum Punkt Recht auf Löschung von Daten

\begin{tabular}{|c|c|}
\hline $\begin{array}{l}\text { Inhaltliche Anpassungen in Patientenunterlagen } \\
\text { nach Forderungen und Hinweisen von Ethik- } \\
\text { kommissionen }\end{array}$ & $\begin{array}{l}\text { Prozessanpassungen als Konsequenz aus den } \\
\text { Inhaltlichen Anpassungen in Patientenunter- } \\
\text { lagen }\end{array}$ \\
\hline $\begin{array}{l}\text { Umsetzung des Rechtes auf Löschung "auf } \\
\text { Verlangen" }\end{array}$ & Umgehende Umsetzung der Datenlöschung \\
\hline $\begin{array}{l}\text { Umsetzung des Rechtes, „wenn die Daten nicht } \\
\text { mehr notwendig sind“" }\end{array}$ & $\begin{array}{l}\text { 1. Überprüfung der Daten und deren Not- } \\
\text { wendigkeit. } \\
\text { 2. Ggf. Hinterlegung des Löschungsgesuches } \\
\text { 3. Löschung wenn Notwendigkeit nicht mehr } \\
\text { gegeben }\end{array}$ \\
\hline "Grundsätzliche" Umsetzung des Rechtes & Umgehende Umsetzung der Datenlöschung \\
\hline $\begin{array}{l}\text { Umsetzung des Rechtes auf Löschung "nach } \\
\text { der gesetzl. Aufbewahrungsfrist" }\end{array}$ & $\begin{array}{l}\text { 1. Hinterlegung des Löschungsgesuches } \\
\text { 2. Löschung nach Fristende }\end{array}$ \\
\hline
\end{tabular}

Bereits eine umgehende Löschung der Daten bei Widerruf stellt eine Prozessanpassung dar (siehe oben, Tab. 3).

Folgende Versionen des Löschungsprozesses nach individueller Meldung bestehen derzeit durch die Formulierungen in Patientenunterlagen:

Eine Umsetzung dieses Rechtes ,auf Verlangen“ kann ebenso individualisiert behandelt werden. Eine andere Formulierung sieht vor, das Recht einzuräumen, die Daten erst zu löschen ,wenn die Daten nicht mehr notwendig sind“, wohingegen sogar die Formulierung der ,grundsätzlichen“ Umsetzung des Rechtes auf Löschung zu finden ist. Diese beiden Optionen widersprechen sich. Auch eine Umsetzung dieses Rechtes „,nach der gesetzlichen Aufbewahrungsfrist“ wird angeboten.

Wird vom Patienten das Recht auf Löschung wahrgenommen, muss derzeit individuell geprüft werden welche Daten zwingend gelöscht werden müssen, wo sich technische und organisatorische Limitierungen ergeben oder ob eine Löschung überhaupt durchgeführt werden darf (unter anderem bei AMG-Studien, wo die Löschung „,nach der gesetzlichen Aufbewahrungsfrist“" greift). In der Regel kann davon ausgegangen werden, dass nach aufwändiger Prüfung jedes Einzelfalles, in den meisten Fällen eine Löschung möglich ist.

\section{Weitergabe von Daten für die Forschung}

Standardmäßig ist für pseudonymisierte medizinische Datensätze mit Einwilligung zur Studienteilnahme und zur weitergehenden biomedizinischen Forschung mit den erhobenen medizinischen Daten eine Datenweitergabe für die Forschung generell möglich und muss nicht gesondert abgefragt werden. Flankierende Maßnahmen wurden implementiert, sodass es sich um eine regulierte Weitergabe auf Antrag nach einem etablierten Auswahl- und Bewertungsprozess handelt. Die Formulierungen zur Weitergabe von Daten und Bioproben 
an Wissenschaftler für Forschungsprojekte haben sich historisch in der Erarbeitungsphase der Antrags- und Auswahlprozesse für die Datennutzung entwickelt.

Die erweiterten Informationspflichten bei Weitergabe von Daten außerhalb der EU sollte für die PICs aller 100 Studienzentren bis zum 25.05.2018 umgesetzt werden. Aufgrund der Use and Access Struktur betrifft dies die klinische Forschungsplattform im Besonderen, weniger jedoch die spezifische klinische Studie. Die bereits beschriebenen einzelnen Abweichungen zwischen und innerhalb der Studien führten dazu, dass die Umsetzung dieser Informationspflichten in Patientenunterlagen nicht zentral durchgeführt werden konnte, sondern von den Studien selbst zu leisten war. Folglich werden zwar in der Regel die nach dem 25.05.2018 einwilligenden Patienten über den Umgang mit der Weitergabe von pseudonymisierten Daten und Biomaterialien außerhalb der EU und außerhalb von Staaten mit Angemessenheitsbeschluss, sowohl mit Sicherheitsmaßnahmen (Standardvertragsklauseln der EU) als auch ohne aufgeklärt. Die Umsetzung, vor allem aus dem frühen Jahre 2018, ähnelt jedoch einem Flickenteppich.

In eine Weitergabe ohne Sicherheitsmaßnahmen/Garantien in Drittländer kann standardmäßig optional eingewilligt werden.

Tab. 5 Auswertung der Inhalte von 75 PICs aus 19 DZHK Studien (Stand Nov 2019) zum Punkt Weitergabe von Daten für die Forschung

\begin{tabular}{|c|c|}
\hline $\begin{array}{l}\text { Inhaltliche Anpassungen in Patientenunterlagen } \\
\text { nach Forderungen und Hinweisen von Ethik- } \\
\text { kommissionen }\end{array}$ & $\begin{array}{l}\text { Prozessanpassungen als Konsequenz aus den } \\
\text { Inhaltlichen Anpassungen in Patientenunter- } \\
\text { lagen }\end{array}$ \\
\hline Weitergabe optional möglich & $\begin{array}{l}\text { Abfrageschritt, ob eine Weitergabe überhaupt } \\
\text { möglich ist, im Prozess zu ergänzen }\end{array}$ \\
\hline a EU+Länder mit Angemessenheitsbeschluss & $\begin{array}{l}\text { Einschränkung auf Weitergabe in Länder der } \\
\text { o.g. Kategorie }\end{array}$ \\
\hline $\begin{array}{l}\text { b EU+Länder mit Angemessenheitsbeschluss } \\
\text { oder Vertrag mit Standardvertragsklauseln }\end{array}$ & $\begin{array}{l}\text { Zusätzlicher Abfrageschritt, ob die Weitergabe } \\
\text { in Fällen unter Einsatz der EU-Standardver- } \\
\text { tragsklauseln möglich, ist zu ergänzen }\end{array}$ \\
\hline c Keine Einschränkungen (immer optional) & $\begin{array}{l}\text { Zusätzlicher Abfrageschritt zu Zeile } 1 \\
\text { (oben), ob die Weitergabe in Drittländer ohne } \\
\text { Garantien möglich, ist zu ergänzen }\end{array}$ \\
\hline
\end{tabular}

Herkunft der Formulierungen:

a „Ausland mit mgl. niedrigerem Datenschutzniveau“ (aus den im Zeitraum der Rekrutierung jeweils aktuellen Musterdokumenten des AKEK übernommen)

b „Dies schließt unter Umständen auch die Weitergabe für Forschungsprojekte in Ländern außerhalb der EU ein. Dies ist generell zulässig, wenn ein Angemessenheitsbeschluss der Europäischen Kommission vorliegt oder behördlich genehmigte Datenschutzklauseln angewendet werden" (neuere Formulierung aus den Musterunterlagen für Biobanken des AKEK, Version 3.0 gemäß Beschluss vom 09.11.2018)

c ,...außerhalb der EU auch in den Fällen zu, in denen kein Angemessenheitsbeschluss der Europäischen Kommission vorliegt und keine behördlich genehmigten Datenschutzklauseln angewendet werden." (neue Formulierung aus Musterunterlagen für Biobanken des AKEK, Version 3.0 gemäß Beschluss vom 09.11.2018) 
Auch zur Weitergabe von Daten für die biomedizinische Forschung wurden die Formulierungen in Patientenunterlagen durch Formulierungs- und Prozessvorgaben von Ethikkommissionen beeinflusst. Daher wurde in einer Studie (alle Studienzentren) sowie in standortspezifischen PIC-Versionen zweier weiterer Studien (einzelne Studienzentren) eine Weitergabe von Daten für die Forschung mit einem eigenen Informationsund Einwilligungsformular abgebildet. In eine Datenweitergabe kann folglich für weitere Forschungsprojekte gesondert eingewilligt und damit auch gesondert widerrufen werden. Diese Widerrufsmöglichkeit kann keine Anonymisierung von Daten nach sich ziehen, da der Datensatz für die Studie weiterhin zur Verfügung stehen muss. Die Einführung dieser Aufteilung stellte daher einen neuen Prozess dar, der zuverlässig implementiert werden musste.

Die Abfrage der Weitergabemöglichkeiten in Drittländer ohne Sicherheitsmaßnahmen/ Garantien von Daten für Forschungsprojekte zog außerdem die Implementierung eines eigenen Abfrageprozesses nach sich, durch den die Spezifizierung der Weitergabemöglichkeiten abgebildet werden kann und Proben nur an entsprechende Partner herausgegeben werden dürfen.

\subsection{Anzahl der Versionen von Einwilligungsunterlagen im DZHK}

Im Rahmen der DZHK-Studien werden für über 100 Studienzentren deutschlandweit (Stand Q4/2019) Einwilligungsunterlagen zentral erfasst, um den Einwilligungsstatus jedes Studienteilnehmers und Biomaterialspenders in Echtzeit vorliegen zu haben. Damit ist gewährleistet, dass Daten und Biomaterialien nur für weitere Forschungsprojekte freigegeben werden, wenn der Patient dem zugestimmt und nicht widerrufen hat.

Für 19 klinische Studien (davon 2 Kohortenstudien), die alle in standardisierter Form und auf Basis der gleichen Grundsätze die klinische Forschungsplattform des DZHK nutzen, wurden von der Treuhandstelle des DZHK mit Stand November 201975 verschiedene elektronische Einwilligungsversionen verwaltet.

Vom Ethik-Projekt des DZHK wurden bis zu diesem Zeitpunkt bereits 122 Einwilligungsversionen erarbeitet, von denen 47 entweder aufgrund von Auflagen oder Hinweisen der Ethikkommissionen bereits vor dem ersten Einsatz überarbeitet wurden und daher nicht zum Einsatz kamen.

\section{$4 \quad$ Beobachtungen und Diskussion}

Unsere Beobachtungen verdeutlichen, dass seit Einführung der DSGVO von Seiten der Ethikkommissionen vermehrt Unsicherheiten bezüglich einer Reihe von datenschutzrechtlichen und sich daraus ergebenden ethischen Fragen bestehen. Alle der Schlagwort-Analyse zugrunde liegenden Fragestellungen von Ethikkommissionen konnten beantwortet werden. Bedenken konnten wir in der Regel ausräumen. Somit war es nach 
mitunter ausführlicher Stellungnahme, bis auf eine Ausnahme, immer möglich, eine gemeinsame Lösung mit der jeweiligen Ethikkommission auszuarbeiten.

Eine Limitierung der Analyse der Ethikvoten ergibt sich zwar durch die Art der Auswertung, die trotz der beschriebenen Kriterien als subjektiv einzustufen ist. Dennoch stellt sich die Frage, ob es gerechtfertigt ist, innerhalb einer klinischen Studie einem Patienten an Standort A andere Informationen und Einwilligungsmodule zur klinischen Forschungsplattform zur Verfügung zu stellen als einem Patienten an Standort B, dem Patienten an Standort C wiederum andere. Der Patient einer anderen Studie am gleichen Standort bekommt wieder andere Informations- und Einwilligungsunterlagen zu diesen Punkten, da die erstberatende Ethikkommission eine andere war. Alle Daten und Biomaterialien werden letztendlich in der gleichen IT-Struktur gespeichert und unter den gleichen streng regulierten Bedingungen für Forschungsvorhaben zur Verfügung gestellt.

Die mit einzelnen Ethikkommissionen ausgearbeiteten Lösungen stellen sich folglich so heterogen dar, dass eine große Anzahl verschiedener Versionen von PICs verwaltet werden muss.

Diese führen zu Änderungen und Ergänzungen in bereits etablierten Prozessen sowie zur Ergänzung neuer Prozesse. Die Einhaltung dieser definierten Prozesse ist essentiell wichtig, um rechtskonform zu arbeiten. Dies betrifft neben Sorgfalts- und Nachweispflichten auch die Umsetzung von Patientenrechten, wie zum Beispiel eine regelkonforme und transparente Durchführung eines Widerrufes. Unklare und sich ändernde Rahmenbedingungen, verstärkt durch das Inkrafttreten der EU-Datenschutzgrundverordnung, führen zu einer Anzahl an Einzellösungen, die die standardisierte Verwaltung und Prozessierung von Daten und Biomaterialien erschwert und viele Ressourcen bindet.

Die Analyse zeigt, wie hoch der Verwaltungsaufwand bereits ist. Bezüglich einzelner Sachverhalte, in der akademischen Forschung unter anderem die Kooperation mit Partnern aus Nicht EU-Drittländern ohne Angemessenheitsbeschluss, stellt sich die Frage, ob die Nachnutzung von medizinischen Daten nicht sogar eklatant behindert, schlimmstenfalls sogar verhindert wird. Im DZHK etablieren sich seit 2019 die ersten Nachnutzungsprojekte, sodass bis heute erst wenige Erfahrungswerte erworben werden konnten. Andere in Deutschland bekannte übergreifende Forschungsplattformen wie die Medizininformatik-Initiative (MI-I), die German Biobank Alliance (GBA) oder Infrastrukturen anderer Deutscher Zentren der Gesundheitsforschung (DZG) agieren entweder in einem anderen Umfeld - wie der Nutzung von klinischen Behandlungsdaten - oder überspannen jeweils nur Teilbereiche der von der klinischen Forschungsplattform des DZHK umfassten Bereiche mit einem zentralen System. Uns sind daher keine derart übergreifenden Plattformen vergleichbarer Größenordnung bekannt.

Für eine öffentlich geförderte Infrastruktur ist es stark erschwerend viele unterschiedliche Prozesse für den gleichen Sachverhalt (z. B. Widerruf) vorzuhalten. Das Akzeptieren eines bereits positiv bewerteten Standardprozesses durch weitere involvierte Ethikkommissionen wäre wünschenswert, um eine standardisierte Durchführung auch in der Zukunft gewährleisten zu können. 
Durch die häufige Notwendigkeit Prozesse anzupassen, ergibt sich eine hohe Komplexität und Vielschichtigkeit unseres IT-Systems. Eine geringe Komplexität dieser würde außerdem Datenschutzaspekte dahin gehend unterstützen, dass die Durchführung jedes Einzelfalles einer Datenlöschung oder eines Widerrufes durchführbar bleibt. So besteht die Gefahr, sich auf die Unverhältnismäßigkeit des Aufwandes (BDSG §35(1)) berufen zu müssen, sofern es nicht mehr möglich wäre, dem Teilnehmerwillen nachgehen zu können indem der Datensatz zu jeder Anfrage adäquat geprüft wird.

Auch in welchem Umfang das Recht auf Löschung dem Patienten für Datenerhebungen im Rahmen der wissenschaftlichen Forschung zu gewähren ist, ist nicht vollständig geklärt. Eine Löschung ist dann vorzusehen, wenn die ,...Daten für die Zwecke, für die sie erhoben oder auf sonstige Weise verarbeitet wurden, nicht mehr notwendig [sind].“ (EU-DSGVO Art.17(1)a). Da sie im Falle einer Nutzung für Forschungsprojekte in einem Forschungsverbund in der Regel noch notwendig wären, gilt BDSG Artikel 17 (3) d. Dazu gehören Archivierungspflichten nach guter klinischer Praxis (GCP) und guter wissenschaftlicher Praxis ebenso wie die Erstellung von BackUps. Auch der kaum zu leistende hohe organisatorische Aufwand einer Löschung im Falle einer umfassenden Nutzung von Datensätzen für verschiedene Forschungsprojekte muss im Sinne der Verhältnismäßigkeit beachtet werden.

Wie kann jedoch einer solchen Zersplitterung einer Dateninfrastruktur, die langfristig angelegt ist, entgegengewirkt werden? Kurzfristige Einzellösungen können nicht das Ziel sein, wenn Forschungsfragestellungen, die einer großen Anzahl medizinischer Datensätze bedürfen, eher zu- als abnehmen. Die pandemische Verbreitung des SARSCoV2 Virus, mit der Notwendigkeit Daten für die medizinische Forschung zeitnah verfügbar zu machen, unterstreicht die Erfordernis stabiler Dateninfrastrukturen für die medizinische Forschung deutlich. Welche Möglichkeiten dafür bietet die EU-DSGVO, welche Möglichkeiten die nationale Umsetzung? Welcher ethische Rahmen sollte für die Nutzung medizinischer Daten angewendet werden und welche Erwartungen hat die Bevölkerung?

Aktuell heißt es einen Konsens zu finden zu Fragen, die nicht nur für das DZHK, wie hier dargestellt, von großer Bedeutung sind. Es betrifft die gesamte akademische Forschungslandschaft, sobald eine standortübergreifende Erhebung und Nutzung von personenbezogenen Daten und Biomaterialien im biomedizinischen Forschungskontext durchgeführt werden soll. Aktuelle Bemühungen wie die der Medizininformatikinitiative zeigen mittlerweile Erfolge im Abstimmungsprozess [MII finalisierte Patienteninformation (Medizininformatik-Initiative 2020)], können aber drängende Fragen wie die datenschutzrechtlich sichere Weitergabe von Daten in Drittländer ohne Angemessenheitsbeschluss der EU, zu denen auch die USA und je nach Empfängerorganisation und Provinz auch Kanada zählen, nicht abschließend beantworten.

Obwohl die EU-DSGVO einen forschungsfreundlichen Ton hat, zeigen unsere Erfahrungen, dass die zuvor weitgehend anerkannte Nutzung des Broad Consent durch Ethikkommissionen und Datenschutzorgane wieder infrage gestellt wird. Auch der 2016 vom AKEK erstellte Mustertext räumte weitreichende Möglichkeiten der breiten 
wissenschaftlichen Nutzung von Bioproben und zugehöriger Daten ein (Mustertext zur Information und Einwilligung in die Verwendung von Biomaterialien und zugehörigen Daten in Biobanken empfohlen vom Arbeitskreis Medizinischer Ethik-Kommissionen (Version 2.0 gemäß Beschluss vom 10.6.2016)).

Doch obwohl die EU-DSGVO mit den Artikeln 5 (b)(e) für wissenschaftliche Forschungszwecke unter gewissen Voraussetzungen, $\mathrm{zu}$ denen explizit die Pseudonymisierung zählt (Art. 89 (1) ${ }^{3}$ ) ein Abweichen von den sonstigen Anforderungen an die Zweckbindung und zeitliche Speicherzeitbegrenzung zur Verarbeitung der Daten akzeptiert (Art. 9(2)j), zeigt unsere Erfahrung eher, dass eine enge Einschränkung auf eingegrenzte Forschungsgebiete innerhalb der Medizin und eine begrenzte Speicherzeit mitunter wieder gefordert wird - in unterschiedlicher Ausprägung. Die DSGVO geht davon aus, dass eine [Weiterverarbeitung... für wissenschaftliche ...Forschungszwecke ...] gemäß Artikel 89 Absatz 1 nicht als unvereinbar mit den ursprünglichen Zwecken (,Zweckbindung“) ... gilt. Warum kann daher nicht wenigstens der Zweck „,medizinische Forschung“ als solcher angegeben werden? Vor allem, da die Literatur eine breite Unterstützung der Bevölkerung untermauert (Richter et al. 2018; Richter et al. 2020; Strech et al. 2016). Es stellt sich gar die Frage, ob ein Einwilligungsformular zur Nutzung von medizinischen Daten und Biomaterialien überhaupt der vom Daten- und Biomaterialspender gewünschte Weg der Legitimation wäre. Oder kann sogar eine Opt Out Lösung in Betracht gezogen werden? Es gibt bereits Belege für eine hohe Akzeptanz dieser Lösung (Richter et al. 2019), so dass diese Option in der künftigen Diskussion zur Umsetzung der medizinischen Forschung mit großen Datenmengen und Biomaterialien mit größerem Interesse betrachtet werden sollte.

Auch scheint unklar, ob dem Daten- und Biomaterialspender die vielfältigen (und unterschiedlichen) Auswahlmöglichkeiten in verschiedenen Patientenunterlagen für eine eigenverantwortliche Datenverwaltung überhaupt von Nutzen sind, da es neben eigenen Erfahrungen auch aus anderen Forschungsverbünden Beispiele gibt, bei denen eine Auswahl einzelner Optionen durch den Teilnehmer nur in den wenigsten Fällen stattfindet (Hoffmann 2018). Die Autoren halten es für dringend notwendig diese Fragen bundesweit einheitlich zu beantworten, um im akademischen Bereich anschlussfähig und finanzierbar zu bleiben. Obwohl auch wir stets individuelle Absprachen mit der jeweiligen Ethikkommission jedes Standortes finden konnten, können Grundsatzfragen auf Basis einer rein juristisch/datenschutzrechtlichen Bearbeitung der Gesetzestexte bis heute nicht vollständig geklärt werden. Trotz der Implementierung zahlreicher Maßnahmen im Rahmen der DZHK Infrastruktur gelingt es in diesem Umfeld nicht, eine weitgehend einheitliche Bewertung für den Einsatz der klinischen Forschungsplattform des DZHK zu erhalten.

Ein erster deutlicher Schritt könnte ein nachhaltiges Vorantreiben des Verfahrens „Koordinierte Bearbeitung multizentrischer Forschungsvorhaben durch die zuständigen Ethik-Kommissionen“ (Ethikkommissionen 2018), initiiert durch den AKEK, sein. Dieses sieht vor, ähnlich dem Verfahren für den Bereich der Klinischen Prüfungen, 
bei denen eine federführende Ethikkommission diese Zuständigkeit übernimmt, eine koordinierende Ethikkommission zu bestellen. Diese orchestriert die berufsrechtliche Bewertung der beteiligten Ethikkommissionen und führt diese zusammen. Bei Teilnahme aller Ethikkommissionen würde dies zu einer deutlichen Erleichterung des organisatorischen Aufwandes für die Datenerhebung und Biomaterialgewinnung in übergreifenden Forschungsplattformen führen.

\section{Literatur}

DZHKe.V. (2017) Klinische Forschungsplattform für Studien. https://dzhk.de/forschung/klinischeforschung-alt/klinische-forschungsplattform. Zugegriffen: 11. Okt. 2020

DZHKe. V. (2018) Transparenz und Fairness bei der Nutzung von Daten und Biomaterial. https:// dzhk.de/forschung/klinische-forschung/nutzung-von-daten-und-biomaterialien-use-and-access. Zugegriffen: 11. Okt. 2020

DZHKe. V. (2019a) Erklärfilm Klinische Forschungsplattform des DZHK. https://www.youtube. $\mathrm{com} /$ watch $? \mathrm{v}=270 \mathrm{VuBvzcj0} \&$ feature $=$ youtu.be. Zugegriffen: 11. Okt. 2020

DZHKe. V. (2019b) Klinische Forschungsplattform (CRP). https://dzhk.de/forschung/klinischeforschung/klinische-forschungsplattform/. Zugegriffen: 11. Okt. 2020

Empfehlung für die Bewertung forschungsbezogener Biobanken durch Ethik-Kommissionen (2016) S 6

Ethikkommissionen AM (2018) Koordinierte Bearbeitung multizentrischer Forschungsvorhaben durch die zuständigen Ethik-Kommissionen. https://www.akek.de/index.php?option=com_cont ent $\&$ view $=$ category $\&$ layout $=$ blog $\& i d=47 \&$ Itemid $=153 \& l a n g=$ de. Zugegriffen: 11. Okt. 2020

Hoffmann WS, D. (2018) Empirische Analyse modularer Einwilligungen: Welche Differenzierungen sind zur Abbildung des Teilnehmerwillens notwendig? In. 10. TMFJahreskongress

Kai K (2020) Art. 17 DS-GVO: Besteht eine Pflicht zur Löschung von Daten aus Backups? In: ZD-Aktuell

Medizininformatik-Initiative (2020) Arbeitsgruppe Consent Mustertext Patienteneinwilligung

Richter G, Borzikowsky C, Lesch W, Semler SC, Bunnik EM, Buyx A, Krawczak M (2020) Secondary research use of personal medical data: attitudes from patient and population surveys in The Netherlands and Germany. Eur J Hum Genet. https://doi.org/10.1038/s41431-02000735-3

Richter G, Borzikowsky C, Lieb W, Schreiber S, Krawczak M, Buyx A (2019) Patient views on research use of clinical data without consent: legal, but also acceptable? Eur J Hum Genet 27(6):841-847. https://doi.org/10.1038/s41431-019-0340-6

Richter G, Krawczak M, Lieb W, Wolff L, Schreiber S, Buyx A (2018) Broad consent for health care-embedded biobanking: understanding and reasons to donate in a large patient sample. Genet Med 20(1):76-82. https://doi.org/10.1038/gim.2017.82

Scheel H, Dathe H, Franke T, Scharfe T, Rottmann T (2019) A privacy preserving approach to feasibility analyses on distributed data sources in biomedical research. Stud Health Technol Inform 267:254-261. https://doi.org/10.3233/shti190835

Strech D, Bein S, Brumhard M, Eisenmenger W, Glinicke C, Herbst T, Jahns R, von Kielmansegg S, Schmidt G, Taupitz J, Troger HD (2016) A template for broad consent in biobank research. Results and explanation of an evidence and consensus-based development process. Eur J Med Genet 59(6-7):295-309. https://doi.org/10.1016/j.ejmg.2016.04.002 
Zeller THC, Umbach N, Franke J, Geier CSH, Knosalla C, Lamp S, Müller AAP, Pickardt T, Schmidt GDT, Troidl C, Weis T, , Krebser JTB, Langner D, Hoffmanna W, Quade MLG, Lee M, Rottmann T, Rienhoff OMK, Wichmann, H-E, Wachter R, Lesser STF, Zimmermann W-H, Nauck M (2014) Central scientific infrastructure for clinical research in the German Centre for Cardiovascular Research - Biobanking and Central Data Management. Vortrag, Biobank Symposium der TMF 2014, Berlin

Open Access Dieses Kapitel wird unter der Creative Commons Namensnennung 4.0 International Lizenz (http://creativecommons.org/licenses/by/4.0/deed.de) veröffentlicht, welche die Nutzung, Vervielfältigung, Bearbeitung, Verbreitung und Wiedergabe in jeglichem Medium und Format erlaubt, sofern Sie den/die ursprünglichen Autor(en) und die Quelle ordnungsgemäß nennen, einen Link zur Creative Commons Lizenz beifügen und angeben, ob Änderungen vorgenommen wurden.

Die in diesem Kapitel enthaltenen Bilder und sonstiges Drittmaterial unterliegen ebenfalls der genannten Creative Commons Lizenz, sofern sich aus der Abbildungslegende nichts anderes ergibt. Sofern das betreffende Material nicht unter der genannten Creative Commons Lizenz steht und die betreffende Handlung nicht nach gesetzlichen Vorschriften erlaubt ist, ist für die oben aufgeführten Weiterverwendungen des Materials die Einwilligung des jeweiligen Rechteinhabers einzuholen.

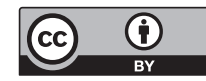

